Questions vives

\section{Questions Vives}

Recherches en éducation

$N^{\circ} 25 \mid 2016$

L'activité des enseignants face au décrochage scolaire : Quelles difficultés et quelles mises en œuvre professionnelles?

\title{
L'activité des enseignants face au décrochage scolaire : Quelles difficultés et quelles mises en œuvre professionnelles?
}

\section{Sylvie Moussay et Éric Flavier}

\section{(2) OpenEdition}

Journals

\section{Édition électronique}

URL : http://journals.openedition.org/questionsvives/1868

DOI : 10.4000/questionsvives.1868

ISSN : $1775-433 X$

\section{Éditeur}

Université Aix-Marseille (AMU)

\section{Édition imprimée}

Date de publication : 7 juillet 2016

ISBN : 978-2-912643-49-0

ISSN : 1635-4079

\section{Référence électronique}

Sylvie Moussay et Éric Flavier, "L'activité des enseignants face au décrochage scolaire : Quelles difficultés et quelles mises en œuvre professionnelles? », Questions Vives [En ligne], N 25 | 2016, mis en ligne le 05 septembre 2016, consulté le 23 septembre 2020. URL : http://journals.openedition.org/ questionsvives/1868; DOI : https://doi.org/10.4000/questionsvives.1868

Ce document a été généré automatiquement le 23 septembre 2020.

\section{(c) (i) (9)}

Questions Vives est mis à disposition selon les termes de la licence Creative Commons Attribution -

Pas d'Utilisation Commerciale - Pas de Modification 4.0 International. 


\title{
L'activité des enseignants face au décrochage scolaire : Quelles difficultés et quelles mises en œuvre professionnelles?
}

\author{
Sylvie Moussay et Éric Flavier
}

Depuis les années 2000, en France, les politiques publiques n'hésitent pas à renforcer les actions de lutte contre le décrochage scolaire et à multiplier les prescriptions institutionnelles (arrêtés, circulaires, référentiel des compétences professionnelles, circulaire de rentrée, etc.) invitant les professionnels à mettre en place des dispositifs spécifiques centrés sur la prévention, la remédiation scolaire, l'orientation réussie (Blaya, 2013; Bruno, Méard \& Walter, 2013). Pour répondre à ces prescriptions, les professionnels se mobilisent et s'engagent dans la conception de dispositifs visant à prendre en charge et à soutenir les élèves à risque de décrochage scolaire ou en rupture professionnelle (Flavier \& Moussay, 2014). L'opérationnalisation de ces dispositifs engendrent des organisations et des collaborations nouvelles entre différents acteurs de l'éducation tant dans le cadre d'alliances éducatives que de la coéducation ou encore d'alternance professionnelle. Son analyse fait apparaître les leviers utilisés par les enseignants et les autres personnels de l'action éducative pour favoriser le processus de raccrochage scolaire ainsi que celui de l'accompagnement après la rupture contractuelle de l'élève. Néanmoins, cette opérationnalisation s'accompagne également de tensions liées aux difficultés professionnelles. Les enseignants expriment parfois le sentiment de ne plus pouvoir agir dans et sur la situation (à risque) de décrochage scolaire. Certains vont même jusqu'à décrire une impuissance à aider les élèves (potentiellement) décrocheurs et une perte de sens attribué à la façon de faire le métier. Dans ce cas, le caractère consubstantiel du décrochage des élèves et du décrochage professionnel permet de souligner la complexité des processus en jeu et montre également toute la pertinence d'une approche collective pour prévenir le décrochage ainsi que pour garantir aux professionnels une posture d'acteurs et non d'agents soumis aux injonctions. 
2 Dans ce contexte, les contributions de ce numéro thématique apportent un éclairage à la fois professionnel et scientifique sur le décrochage en accordant un intérêt particulier au travail réel des acteurs impliqués. En référence à des ancrages théoriques divers, chaque étude se structure autour d'un axe spécifique mettant en avant une approche originale du processus de décrochage

3 Le premier axe s'intéresse à l'activité des professionnels (enseignants, personnels de direction, conseiller principal d'éducation, éducateurs, etc.) engagés dans les dispositifs de lutte contre le décrochage scolaire. La compréhension de cette activité s'appuie sur des observations in situ mais aussi sur des entretiens avec les professionnels qui expriment notamment le sens accordé au travail avec les élèves décrocheurs. En s'inscrivant dans cet axe, les textes permettent ainsi de saisir les circonstances dans lesquelles les professionnels prennent en charge collectivement la problématique du décrochage au travers d'outils et de dispositifs. L'étude présentée par Jacques Méard vise à mieux comprendre comment les enseignants d'Education physique prennent en compte le processus de persistance ou de désengagement des élèves qui conduisent, dans certains cas, à des «dispenses ». Dans la même veine, la compréhension de l'activité des professionnels œuvrant cette fois-ci au sein d'un dispositif d'accrochage est au centre de l'étude de Chantal Tièche Christinat, Valérie Angelucci et de Léonie Liechti. Ces auteures interrogent la nature des leviers pédagogiques que les professionnels actionnent pour atteindre les objectifs d'accrochage. Dans un autre contexte d'enseignement, celui d'un collège en zone d'éducation prioritaire, Sylvie Moussay et Véronique Blanjoie décrivent leur intervention-recherche accompagnant un collectif d'enseignants dans l'analyse de leur travail pour leur permettre d'agir sur les situations de décrochage et de transformer collectivement leurs modalités d'action face aux élèves décrocheurs. Eric Flavier étudie pour sa part l'activité d'un collectif d'enseignants engagés dans un dispositif de prévention du décrochage scolaire, «l'entreprise virtuelle». Son étude montre précisément comment ce dispositif amène les enseignants vers une nouvelle professionnalité selon une dynamique d'activité collective caractérisée par l'intrication et l'interdépendance de leurs actions respectives en soutien des élèves à risque de décrochage.

4 Le deuxième axe souligne les effets du décrochage des élèves sur l'activité de travail des enseignants. Il interroge ainsi les tensions liées aux difficultés pour les enseignants de travailler avec les élèves décrocheurs et de faire face à l'imprévisibilité accrue de la situation d'enseignement-apprentissage liée à la présence de ces élèves au sein du cours, comme le montre précisément Françoise Bruno dans son étude se référant à une approche développementale de l'activité. Les tensions sont souvent à l'origine d'une crise identitaire, d'isolement voire d'abandon des professionnels. L'enquête réalisée par Séraphin Alava propose d'identifier cette crise à travers une échelle du décrochage enseignant en lien avec les formes d'épuisement et de mal-être de l'enseignant. Dans le prolongement de ces deux études, les résultats du travail de recherche de Philippe Zimmermann questionnent le lien entre les processus d'accrochage et de décrochage professionnel des enseignants novices face aux élèves décrocheurs. Son étude plaide pour une formation des enseignants à la prise en compte du risque de décrochage sur le plan de l'efficience, dans une démarche de conception de nouveaux outils.

5 Le troisième axe s'intéresse à la dynamique du décrochage et aux conditions dans lesquelles les élèves passent progressivement d'un état de désengagement voire de rupture scolaire à celui d'engagement ou de reprise dans le travail scolaire. Stéphane 
Guillon et Stéphanie Hinsinger montrent ainsi que la rupture de contrat, en l'appréhendant comme un événement marquant le parcours de formation de l'apprenti, peut être évitée par une préparation à l'alternance et un accompagnement favorisant l'orientation scolaire et les relations professionnelles avec le maître d'apprentissage. Liliane Pelletier et Driss Alaoui s'intéressent à la dynamique de décrochageraccrochage scolaires de collégiens engagés sur une durée de quatre mois dans un dispositif de soutien à la persévérance scolaire. Ils mettent en exergue les microtransformations chez les élèves dans le rapport à soi et à autrui ainsi que dans le rapport à l'école, aux enseignants, aux pairs et à la famille. Enfin, l'étude présentée par Olivier Vors décrit le décrochage local, en cours d'éducation physique et sportive, à partir de l'analyse des préoccupations des élèves, de leurs perceptions et des connaissances mobilisées dans l'action. A partir des matériaux recueillis lors des entretiens, l'étude montre que l'activité des élèves est structurée autour d'un faisceau de préoccupations centrées sur le travail et le jeu et comprenant une dynamique d'alternance de « micro-phases » de décrochage et de raccrochage des élèves au travail scolaire.

\section{AUTEURS}

\section{SYLVIE MOUSSAY}

Maître de conférences, École supérieure du professorat et de l'éducation (ESPE), Université Clermont-Auvergne

\section{ÉRIC FLAVIER}

Maître de conférences, École supérieure du professorat et de l'éducation (ESPE), Université de Strasbourg 